\title{
Experimental Investigation of Laser Surface Hardening of AISI 4340 Steel Using Different Laser Scanning Patterns
}

\author{
Baha Tarchoun 1 , Abderrazak El Ouafi ${ }^{*}$, Ahmed Chebak ${ }^{1,2}$ \\ ${ }^{1}$ Department of Engineering, University of Quebec at Rimouski, Rimouski, Canada \\ ${ }^{2}$ Innovation Lab for Operations, Mohammed VI Polytechnic University, Benguerir, Morocco \\ Email: *Abderrazak_elouafi@uqar.ca
}

How to cite this paper: Tarchoun, B., El Ouafi, A. and Chebak, A. (2020) Experimental Investigation of Laser Surface Hardening of AISI 4340 Steel Using Different Laser Scanning Patterns. Journal of Minerals and Materials Characterization and Engineering, 8, 9-26.

https://doi.org/10.4236/jmmce.2020.82002

Received: January 9, 2020

Accepted: March 7, 2020

Published: March 10, 2020

Copyright $\odot 2020$ by author(s) and Scientific Research Publishing Inc. This work is licensed under the Creative Commons Attribution International License (CC BY 4.0).

http://creativecommons.org/licenses/by/4.0/

\begin{abstract}
Laser surface transformation hardening becomes one of the most modern processes used to improve fatigue and wear properties of steel surfaces. In this process, the material properties and the heating parameters are the factors that present the most significant effects on the hardened surface attributes. The control of these factors using predictive modeling approaches to achieve desired surface properties leads to conclusive results. However, when the dimensions of the surface to be treated are larger than the cross-section of the laser beam, various laser-scanning patterns are involved. This paper presents an experimental investigation of laser surface hardening of AISI 4340 steel using different laser scanning patterns. This investigation is based on a structured experimental design using the Taguchi method and improved statistical analysis tools. Experiments are carried out using a $3 \mathrm{~kW} \mathrm{Nd:} \mathrm{YAG}$ laser source in order to evaluate the effects of the heating parameters and patterns design parameters on the physical and geometrical characteristics of the hardened surface. Laser power, scanning speed and scanning patterns (linear, sinusoidal, triangular and trochoid) are the factors used to evaluate the hardened depth and the hardened width variations and to identify the possible relationship between these factors and the hardened zone attributes. Various statistical tools such as ANOVA, correlations analysis and response surfaces are applied in order to examine the effects of the experimental factors on the hardened surface characteristics. The results reveal that the scanning patterns do not modify the nature of the laser parameters' effects on the hardened depth and the hardened width. But they can accentuate or reduce these effects depending on the type of the considered pattern. The results show also that the sinusoidal and the triangular patterns are relevant when a maximum hardened width with an acceptable hardened depth is desired.
\end{abstract}




\section{Keywords}

Laser Surface Hardening Process, Experimental Investigation, Laser Scanning Patterns, AISI 4340 Steel, Hardness Profile, Hardened Depth, Hardened Width, Design of Experiment, Analysis of Variance

\section{Introduction}

Laser surface transformation hardening process (LSTHP) is increasingly used to heat treat mechanical parts surfaces in order to enhance their mechanical properties. By applying concentrated, short and localized thermal inputs, LSTHP can improve the wear and fatigue resistance of the treated zones [1] [2]. Compared to other surface heat treatment processes, LSTHP presents several advantages such as selective and precise hardening zones, minimization of undesirable deformation and distortion, application of very fast thermal cycles to obtain autogenous hardening, treatment for parts with complex geometry through the use of robots, and possibility of full process automation and control [3] [4].

In LSTHP, the hardened surface characteristics depend essentially on the laser heating process parameters and the thermo-physical properties of the material to be treated. For an adequate use of this process, it is necessary to better understand the operating mode and to develop robust predictive models that can be used to control and adjust the process parameters in order to estimate precise hardened surface characteristics without using the traditional long and costly experimental procedures [3] [4] [5]. The produced models can be used to predict the hardness profile attributes for various heating parameters and conditions. Two kinds of models can be developed in this perspective: numerical models and empirical models. The numerical models combine laser spot form and motion, non-linear properties of the material and the heat flow equation using the finite element method (FEM). This approach uses a heat flow equation in order to determine the temperature distribution for each heat treatment phase. The hardness is then approximated by the Ashby and Easterling equations [3] [6]. The empirical models are based on experimental data that can be collected using structured design of experiments, and analyzed using statistical tools in order to extract the arithmetical relationship between the heating parameters and the hardness profile attributes.

In LSTHP of large surfaces, the simplest technique frequently used is called multi-track treatment. This technique consists to execute several passes one next to the other until the surface is completely covered. In some cases, the number of laser beam passes can be prohibitive. The reduction of the number of passes for a given surface can be, theoretically, achieved by increasing the diameter of the beam spot. This is an unrealistic and very expensive solution. In addition, the multi-track technique generates overheated zones between every two successive passes where the hardness decreases dramatically. Therefore, it is necessary to 
improve the technique or to look for other alternatives [7] [8]. Researchers such as Lakhkar et al. have improved this method while trying to minimize overheated areas between hardening tracks where hardness decreases, and have succeeded to obtain a hardness that varies between 48 and 58 HRC using $850 \mathrm{~W}$ laser power. They have found also that hardness variation can be controlled by the spacing between the tracks [7]. But this method is still unsatisfactory since the number of passes increases rapidly by increasing the surface to be treated, which prolongs the treatment time and increases the energy supply causing the deformation of parts. Xue et al. [9] [10] have worked on another treatment technique called "Dot matrix treatment", which generally consists of treating just a certain percentage of the surface with a uniform distribution using a pulsed laser beam. This method has reduced the energy applied to the part to be treated, thus avoiding distortion of the part, but the risk of wear and tear in untreated areas always precedes the treated areas [9] [10]. Using this method, Straffelini et al. proved that tensile strength and wear resistance increased as the density of the matrix increased [11].

There is also another interesting treatment technique that can be used and which is based on the use of complex laser beam moving patterns during the laser hardening process. The use of certain types of trajectories allows mainly to treat the maximum of the surface with a single pass, this allows to optimize the laser treatment process of the relatively large geometries. To date, few pieces of research have addressed this method although the energy supply is enormously optimized and the treatment time is reduced. Sistaninia et al. are almost among the only researchers who have studied this method of treatment [12]-[17]. However, their efforts were focused on the development of analytical models that analyze the temperature distribution obtained by different beam displacement patterns, which explains the importance of an experimental analysis of the effect of this technique on the obtained hardness profile. Sistaninia and Farrahi have determined that for given laser power, a sinusoidal moving trajectory has a more uniform temperature distribution than a trochoid trajectory [12] [16], and Zhou et al. have shown that an increase in frequency leads to a drop in the maximum temperature [13] [17]. Therefore, an experimental investigation of interactions between the different phenomena during the hardening process is necessary to develop predictive models able to control the parameters of this heat treatment technique in order to produce the appropriate hardened surface characteristics.

The objective of this paper is to evaluate the effect of using different displacement trajectories during the laser heat treatment process of AISI 4340 steel plates. This is done by conducting an experimental investigation of the moving pattern effect and the process parameters' effect on the hardness profile. This study allows to determine the trajectory that will allow to have the best hardness profile (best depth and hardness distribution) to optimize the process. The investigation is based on a structured design of experiments and various statistical analysis tools. Experiments are carried out by using a $3 \mathrm{~kW} \mathrm{Nd:} \mathrm{YAG} \mathrm{laser}$ source where four moving patterns are considered: linear, sinusoidal, triangular 
and trochoid pattern. The obtained results permit to understand the effects of patterns as well as laser parameters on the hardened zone attributes.

\section{Experimentation}

\subsection{Methodology}

The experimental investigation is conducted on AISI 4340 steel $60 \mathrm{~mm}$ long, 6 $\mathrm{mm}$ wide, $5 \mathrm{~mm}$ thick plates hardened and tempered to 25 - $30 \mathrm{HRC}$ (Figure 1). The parts were oil quenched after complete austenitization in the furnace, then quenched to blacken their color and to improve the absorptivity of the laser irradiation. The chemical composition of the material is shown in Table 1. To carry out the various experiments, an Nd: YAG laser source was used, powered by a YLS-3000-ST2 IPG fiber laser and mounted on a six-axis FANUC M-710IC robot. The ILVDC-Scanner system mounted on the laser head is used to perform the desired scanning patterns. The used laser source has a maximum power of $3000 \mathrm{~W}$, a wavelength of $1070 \mathrm{~nm}$ and a focal length of $310 \mathrm{~mm}$. The diameter of the focal spot used in this study is $0.52 \mathrm{~mm}$, and it can be modified by means of a collimator fixed on the laser head. The main elements of the laser cell are presented in Figure 2. Figure 3 illustrates the four moving patterns considered in this study.

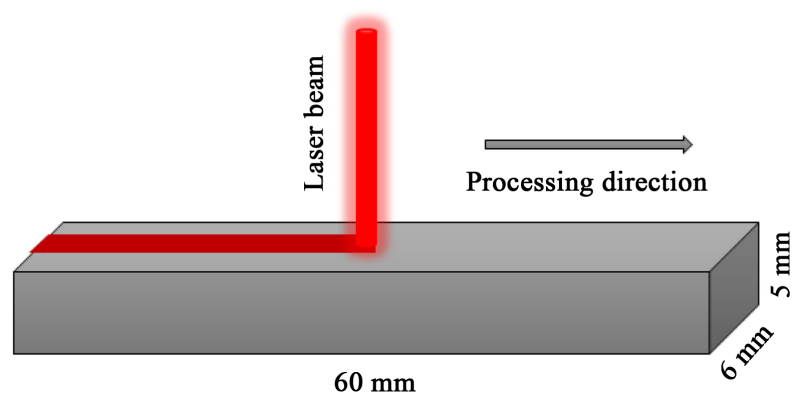

Figure 1. A schematic illustration of laser transformation hardening.

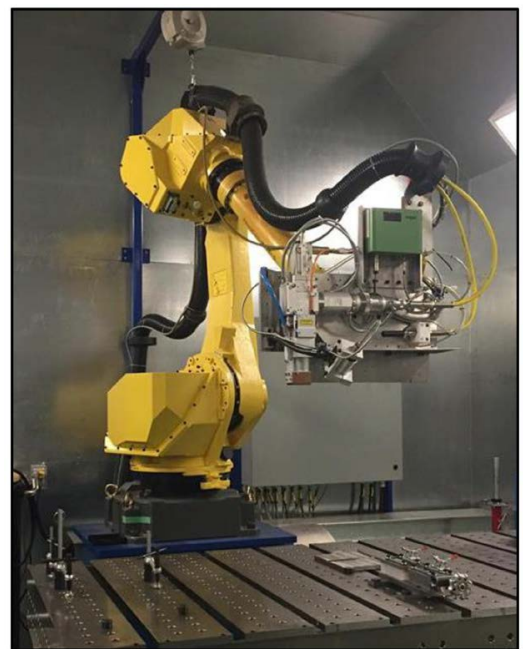

Figure 2. Experimental setup of steel plate's laser surface hardening using laser cell. 

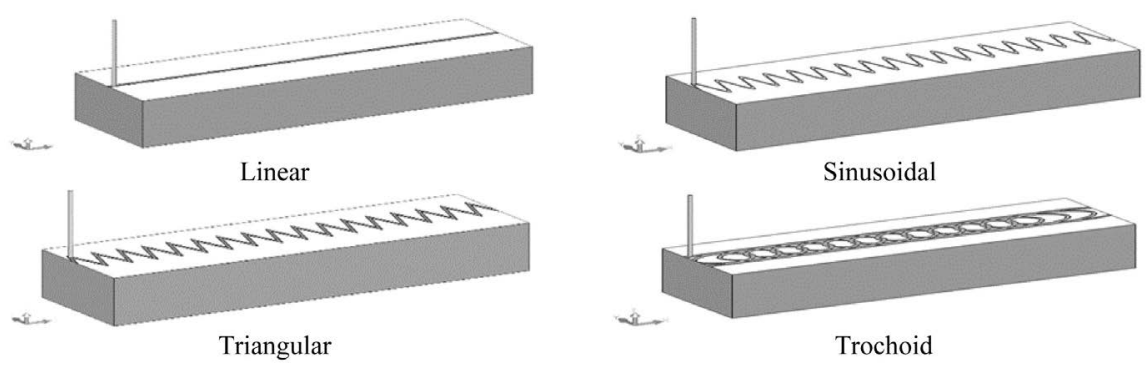

Figure 3. Schematic illustration of the considered laser scanning patterns.

Table 1. Chemical composition of AISI 4340 steel.

\begin{tabular}{cccccccccccc}
\hline Element & $\mathrm{Fe}$ & $\mathrm{Ni}$ & $\mathrm{Cr}$ & $\mathrm{Mn}$ & $\mathrm{C}$ & $\mathrm{Mo}$ & $\mathrm{Si}$ & $\mathrm{S}$ & $\mathrm{P}$ & $\mathrm{Ce}$ & $\mathrm{Cf}$ \\
\hline$\%$ & 95.71 & 1.83 & 0.81 & 0.7 & 0.4 & 0.25 & 0.23 & 0.04 & 0.03 & 0.8 & 0.01 \\
\hline
\end{tabular}

The main parameters selected in the present study are laser power (P), Scanning speed (SS) and type of moving pattern. As shown in Figure 4, the measured quantities are the hardened depth (HD) and the hardened width (HW), which represent two properties to be optimized by the laser heat treatment process. After the laser processing, the samples are analyzed using a standard metallographic approach that consists of carefully cutting the plates using an Abrasi-Matic300 cutting machine, then coating and polishing the samples, then measuring the hardness of the parts and finally performing the chemical etching to measure the hardened depth and the hardened width. The chemical attack was performed using Nital's chemical solution (95\% ethanol and 5\% nitric acid). The hardened depth and hardened width measurements are taken by a CLEMEX precision microscope, and the hardness profile is determined based on the micro-hardness measurements using the Clemex machine.

\subsection{Preliminary Tests}

Due to the lack of a database in the literature that provides the ranges of variation of the laser hardening process parameters, preliminary tests were carried out to define its intervals in order to ensure a hardened surface with a minimal melting zone. By varying one parameter at a time, the combination of maximum power and minimum scanning speed allows to have maximum hardened depth and maximum hardened width but with some surface melting. On the other hand, the combination of minimum power and maximum scanning speed allows to have a minimum hardening depth and width but without melting the surface. Consequently, the minimum and maximum limits for each processing parameter are defined based on these preliminary tests, where the variation range of the power is selected between 500 and $800 \mathrm{~W}$ and the speed varies between 20 and $40 \mathrm{~mm} / \mathrm{s}$.

\subsection{Experimental Design}

To establish experimental tests, it is essential to use an experimental design that 


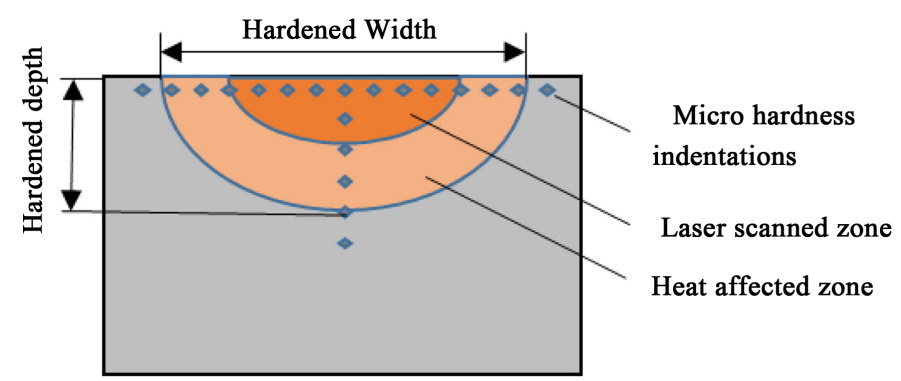

Figure 4. Cross-section of the steel plate with the variables to be measured.

can provide relevant results with the minimum number of tests to be performed, while respecting the ranges of variation already defined by the preliminary tests. Depending on the number of factors and their assigned levels, a systematic design based on factor design is an excellent tool to use and leads to an understanding of the material's behavior towards this processing technique. Due to their efficiency and simplicity, factorial designs are the most advised recommended designs to use, although the number of tests increases exponentially when an additional parameter or level is adopted at design, making the study more expensive and time-consuming [18]. However, fractional designs can collect statistically significant data on the studied phenomenon, on the effects of the process parameters and even on the interactions between the factors with the minimum number of tests [18]. Consequently, a Taguchi plan was established as an experimental design for this study. For each moving trajectory, 2 factors, 3-levels $\mathrm{L}_{9}$ matrix is used in order to determine the effect of power and scanning velocity on the hardened depth and hardened width of the steel plates. Indeed, the choice to introduce three levels for each parameter is intended to study the nonlinearity of the parameter effects and the interactions between them. The selected factors and their levels are identified in Table 2. The experimental design that best fit this problem is illustrated in Table 3.

\subsection{Preliminary Tests and Repeatability}

To improve the accuracy and validity of the results, four tests were performed under the same working conditions (power of $650 \mathrm{~W}$ and a scanning speed of 30 $\mathrm{mm} / \mathrm{s}$ for a linear track), which also allows to estimate the overall and relative errors of measurements. These errors can be caused by uncontrollable factors such as a sudden change in ambient temperature during processing, non-uniformity of the chemical composition of the base material, incorrect calibration or use of measuring instruments, etc. The results presented in Table 4 show good precision of the measurement since the variations are less than $6 \%$. These results then guarantee the validity of the measurement method and make it possible to carry out the final tests with confidence.

\section{Results Analysis and Discussion}

The use of different laser scanning patterns leads to different hardened surfaces 
Table 2. Selected parameters and their levels for experimentation.

\begin{tabular}{cccc}
\hline Factor & Level 1 & Level 2 & Level 3 \\
\hline Power $(\mathrm{W})$ & 500 & 650 & 800 \\
Scanning speed $(\mathrm{mm} / \mathrm{s})$ & 20 & 30 & 40 \\
\hline
\end{tabular}

Table 3. Experimental design used for each laser scanning patterns.

\begin{tabular}{ccc}
\hline Tests & Power $(\mathrm{W})$ & Scanning speed $(\mathrm{mm} / \mathrm{s})$ \\
\hline 1 & 500 & 20 \\
2 & 500 & 30 \\
3 & 500 & 40 \\
4 & 650 & 20 \\
5 & 650 & 30 \\
6 & 650 & 40 \\
7 & 800 & 20 \\
8 & 800 & 30 \\
9 & 800 & 40 \\
\hline
\end{tabular}

Table 4. Results of the repetition tests.

\begin{tabular}{ccccc}
\hline & Max & Min & Mean & Relative error \\
\hline HD $(\mu \mathrm{m})$ & 665 & 626 & 653.75 & $5.86 \%$ \\
HW $(\mu \mathrm{m})$ & 865 & 838 & 854 & $3.12 \%$ \\
\hline
\end{tabular}

topology in terms of hardened depth, hardened width and hardness homogeneity. A slight surface fusion is also present in some cases but in a negligible and acceptable gradation, since the selection of parameter variation ranges during the preliminary tests contributed significantly to minimize this phenomenon. Figure 5 shows typical hardness profiles achieved using various laser parameters applied to the traditional linear scanning pattern. Images $5 \mathrm{a}, 5 \mathrm{~b}$ and $5 \mathrm{c}$ present the hardness profiles achieved using scanning velocity of $20 \mathrm{~mm} / \mathrm{s}$ for power of 500,650 and $800 \mathrm{~W}$ respectively. Images $5 \mathrm{~d}, 5 \mathrm{e}$ and $5 \mathrm{f}$ illustrate the hardness profiles at a fixed power of $800 \mathrm{~W}$ and at scanning speed of 20,30 and $40 \mathrm{~mm} / \mathrm{s}$, respectively. Figure 5(a) illustrates also the difference between the base metal and the hardened zone at the microscopic level where it is clear that the metallographic structure of the treated area is denser than that of the base metal. This density is the result of the immigration of carbon grains into the austenite during heating by the laser beam and its transformation into martensite during cooling. From these figures, it is clear as expected that the laser power has a positive effect on the hardness zone dimensions, while the increase of the scanning velocity has a negative effect. These observations confirm that both factors have direct effects on the hardness zone in terms of both hardened depth and hardened width. However, these effects that are the consequences of spatial 


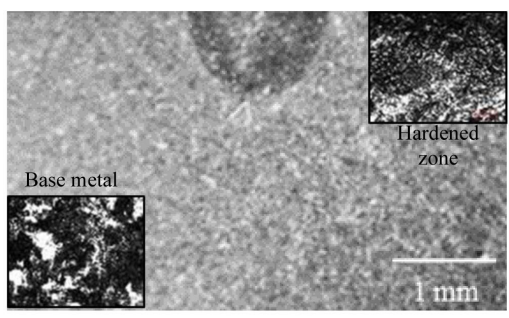

(a)

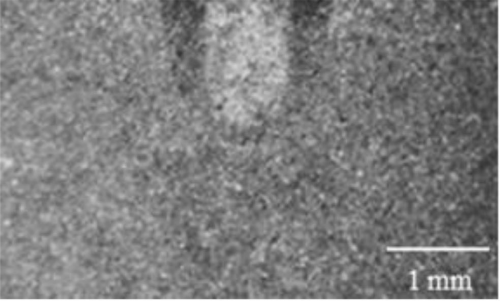

(c)

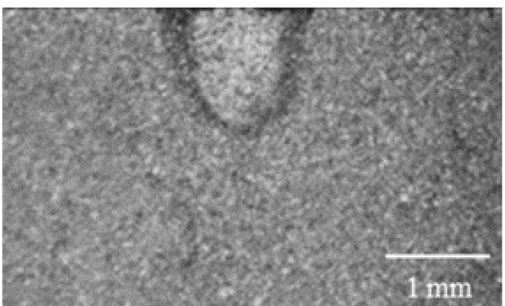

(e)

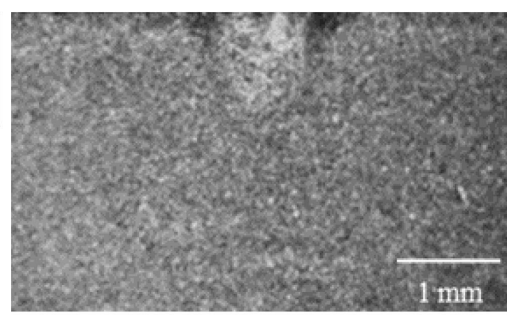

(b)

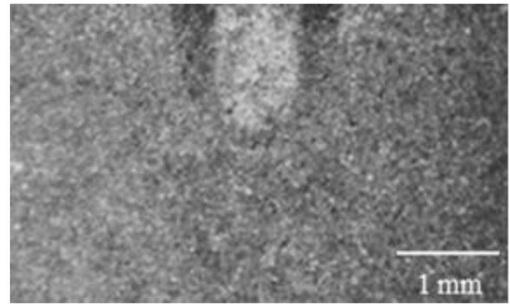

(d)

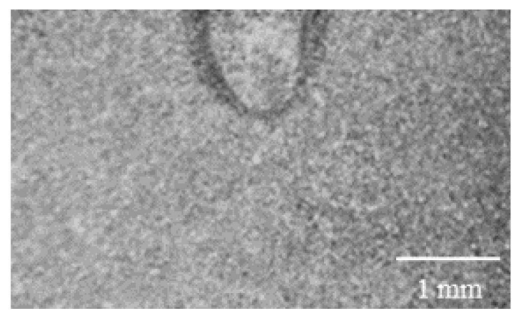

(f)

Figure 5. Typical hardened zones achieved using linear scanning pattern at various laser parameters.

temperature distribution can be completely transformed when using others scanning patterns. This transformation modifies considerably the thermal topology of the hardened surface.

Figure 6 shows typical hardness profiles achieved using various laser scanning patterns. The variation of HD and HW from a scanning pattern to another is directly related to pattern specifications such as amplitude, frequency and focal spot. The figure describes the variation of hardness profile for test 7 that uses a scanning velocity of $20 \mathrm{~mm} / \mathrm{s}$ and laser power of $800 \mathrm{~W}$. The scanning pattern geometry affects the exposure time of different portions of the part surface to the laser radiation. Depending on the scanning pattern, some zones of the surface can be exposed to the heat source repeatedly and for a longer time. An increase of the exposure interval gives more time for the carbon to migrate homogeneously into the austenite producing a larger treated surface. However, this time must not exceed certain limits to avoid melting zones and unwanted deformations of the treated surface.

Moreover, Figure 6 shows that the sinusoidal pattern presents the best compromise between hardened depth, hardened width and hardness distribution uniformity. Trochoid pattern produces high HD and HW but with a very irregular hardness distribution. The triangular pattern produces HD and HW with a uniform distribution but remains less than that achieved by sinusoidal pattern. 


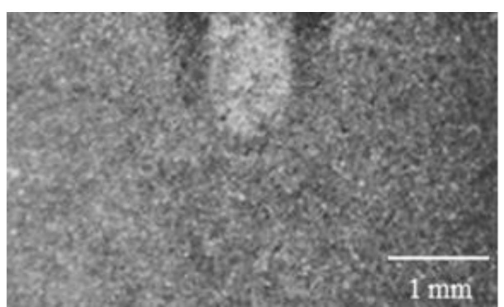

(a)

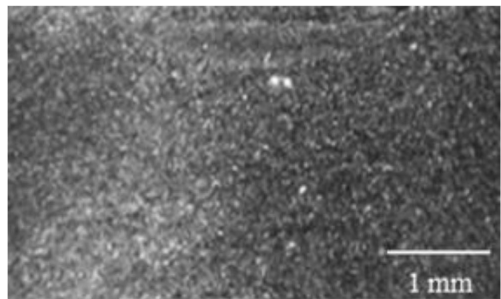

(c)

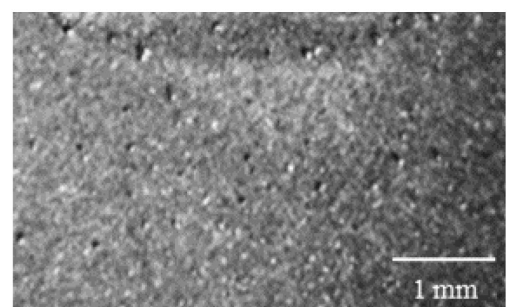

(b)

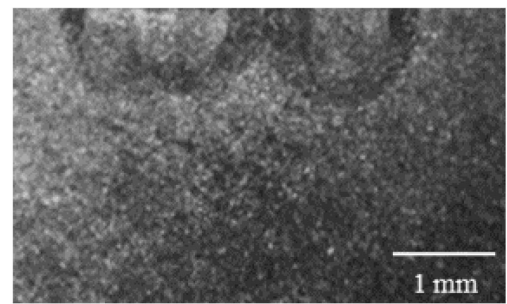

(d)

Figure 6. Typical hardened zones using a power of $800 \mathrm{~W}$ and a scanning speed of 20 $\mathrm{mm} / \mathrm{s}$ for linear, sinusoidal, triangular and trochoid scanning patterns respectively.

Linear pattern presents, as anticipated, the maximum hardened depth and a good hardness distribution but with the lowest HW. In the linear scanning pattern, the laser power remains concentrated below the focal spot. HD is maximum but HW slightly exceeds the beam diameter. For the other patterns, the laser power is distributed over the entire amplitude of the trajectory increasing the width of the hardened surface. The increase of the HW results inexorably in a decrease of HD.

Table 5 presents the hardened depths and hardened widths collected from the experiments. These results are analyzed using several statistical indicators extracted from the analysis of variance (ANOVA) such as percent contribution of each parameter in HD and HW variations, the average effects graphs, the correlation between the measured characteristics and the laser parameters, and the graphs of the response surfaces. Generally, the parameter with the highest percentage contribution represents the most significant parameter. The total sum of the contributions of the considered parameters should reach $100 \%$. The difference is usually attributed to uncontrolled factors or experimental errors.

The graphs of average effects consist of visualizing the effect of each parameter on the hardened zone characteristics. These graphs allow easy comparisons between the parameter's effects and a better understanding of the connections between these parameters and the characteristics to evaluate. The response surface method (RSM) is adopted to explore the nature of relationships between the experimental factors and the hardness profiles.

The results of ANOVA performed on the four $\mathrm{L}_{9}$ are presented in Table 6 . The analysis of the parameter contribution reveals different results between linear pattern and other scanning patterns. Generally, the laser power affects HD more than the scanning speed regardless of the scanning pattern. However, the scanning speed affects HW more than the power. An increase in power 
Table 5. Experimental results achieved using the four performed $\mathrm{L}_{9}$.

\begin{tabular}{ccccccccc}
\hline \multirow{2}{*}{ Tests } & \multicolumn{2}{c}{ Linear pattern } & \multicolumn{2}{c}{ Sinusoidal pattern } & \multicolumn{2}{c}{ Triangular pattern } & \multicolumn{2}{c}{ Trochoid pattern } \\
\cline { 2 - 8 } & HD $(\mu \mathrm{m})$ & $\mathrm{HW}(\mu \mathrm{m})$ & $\mathrm{HD}(\mu \mathrm{m})$ & $\mathrm{HW}(\mu \mathrm{m})$ & $\mathrm{HD}(\mu \mathrm{m})$ & $\mathrm{HW}(\mu \mathrm{m})$ & $\mathrm{HD}(\mu \mathrm{m})$ & $\mathrm{HW}(\mu \mathrm{m})$ \\
\hline $\mathbf{1}$ & 623 & 946 & 263 & 2708 & 259 & 2486 & 613 & 2425 \\
$\mathbf{2}$ & 458 & 854 & 167 & 2550 & 180 & 2405 & 593 & 2341 \\
$\mathbf{3}$ & 392 & 755 & 125 & 2293 & 147 & 2312 & 457 & 2262 \\
$\mathbf{4}$ & 809 & 984 & 330 & 2779 & 490 & 2504 & 632 & 2493 \\
$\mathbf{5}$ & 661 & 862 & 243 & 2607 & 280 & 2436 & 619 & 2384 \\
$\mathbf{6}$ & 587 & 778 & 189 & 2478 & 224 & 2354 & 482 & 2302 \\
$\mathbf{7}$ & 1011 & 1154 & 456 & 2808 & 574 & 2516 & 802 & 2578 \\
$\mathbf{8}$ & 894 & 1027 & 308 & 2684 & 426 & 2483 & 757 & 2410 \\
$\mathbf{9}$ & 820 & 945 & 251 & 2507 & 306 & 2368 & 713 & 2359 \\
\hline
\end{tabular}

Table 6. ANOVA analysis for the hardened depth and width for the four moving patterns.

\begin{tabular}{|c|c|c|c|c|c|}
\hline \multirow{2}{*}{ Pattern } & \multirow{2}{*}{ Source } & \multicolumn{2}{|l|}{$\mathrm{HD}$} & \multicolumn{2}{|l|}{ HW } \\
\hline & & Contribution (\%) & F-value & Contribution (\%) & F-value \\
\hline \multirow{4}{*}{ Linear } & Power & 78.20 & 20.09 & 42.91 & 2.22 \\
\hline & Scanning speed & 20.69 & 7.10 & 48.33 & 0.62 \\
\hline & Power ${ }^{\star}$ SS & 0.12 & 0.61 & 0.06 & 0.04 \\
\hline & Error & 0.99 & - & 8.70 & - \\
\hline \multirow{4}{*}{ Sinusoidal } & Power & 45.27 & 13.46 & 15.61 & 0.05 \\
\hline & Scanning speed & 50.11 & 0.03 & 80.43 & 16.81 \\
\hline & Power ${ }^{*}$ SS & 1.44 & 2.27 & 1.52 & 3.09 \\
\hline & Error & 3.18 & - & 2.45 & - \\
\hline \multirow{4}{*}{ Triangular } & Power & 51.15 & 16.91 & 10.42 & 0.10 \\
\hline & Scanning speed & 41.18 & 0.56 & 86.31 & 9.50 \\
\hline & Power ${ }^{*}$ SS & 3.60 & 4.43 & 0.39 & 0.68 \\
\hline & Error & 4.07 & - & 2.87 & - \\
\hline \multirow{4}{*}{ Trochoid } & Power & 58.20 & 0.31 & 22.65 & 7.73 \\
\hline & Scanning speed & 32.48 & 1.14 & 73.07 & 0.52 \\
\hline & Power ${ }^{*}$ SS & 1.06 & 0.32 & 1.05 & 1.62 \\
\hline & Error & 8.26 & - & 3.23 & - \\
\hline
\end{tabular}

mainly leads to an increase in HD and HW, while an increase in the scanning speed leads to a decrease in HW and a decrease in HD.

For linear pattern, power contribution represents more than $75 \%$ in the HD variation while it varies between $45 \%$ and $58 \%$ for the rest of the patterns. The 
contribution of the scanning speed in the $\mathrm{HW}$ variation is between $73 \%$ and $86 \%$ for sinusoidal, triangular and trochoid patterns. However, it does not exceed $50 \%$ for linear pattern. The contribution of the error is minimal in most cases and does not exceed $10 \%$. This points out the accuracy of the achieved experimental results and presumes good experimental planning and measurement.

These observations are confirmed by the graphs of the average effects presented in Figures 7-10. According to these figures, it is clear that the increase of laser power affects positively the hardened depth and width, while the increase of scanning speed affects negatively these two characteristics for the four scanning patterns. The magnitude of these effects varies considerably from one pattern to another. These results are confirmed by the correlation analysis between laser parameters and hardened zone dimensions for the four scanning patterns presented in Table 7. Strong correlation can be observed between power and HD and between scanning speed and HW.
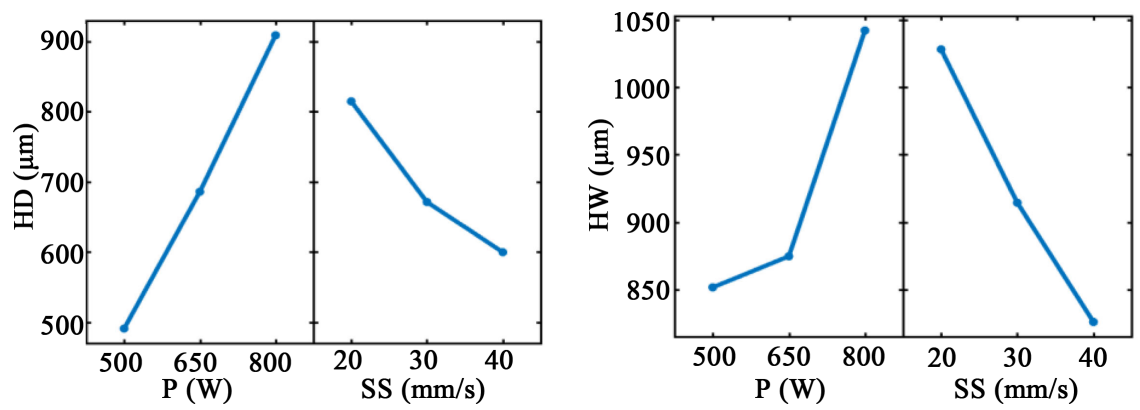

Figure 7. Main effects of laser parameters on HD and HW for the linear moving pattern.
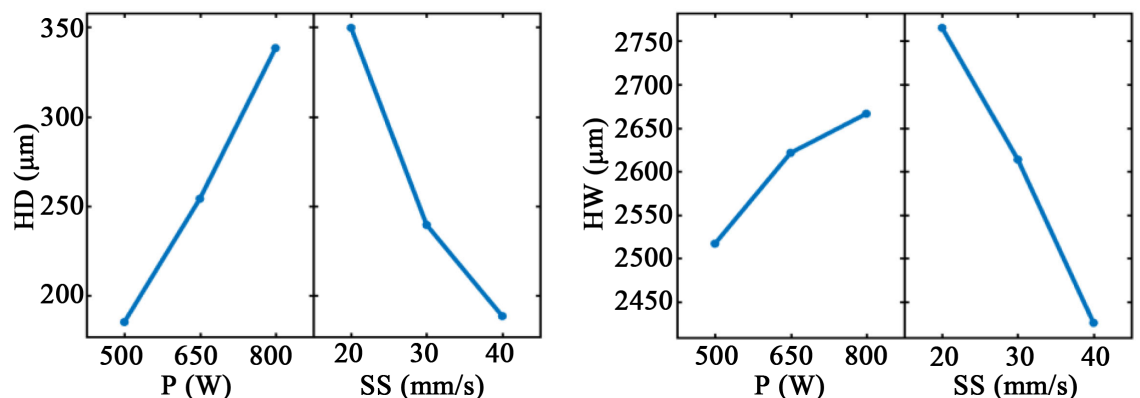

Figure 8. Main effects of laser parameters on HD and HW for sinusoidal moving pattern.
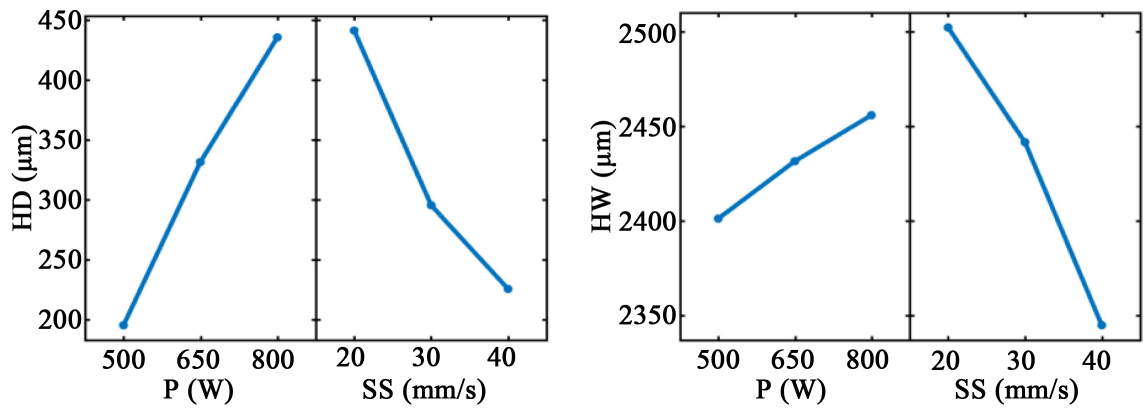

Figure 9. Main effects of laser parameters on HD and HW for triangular moving pattern. 

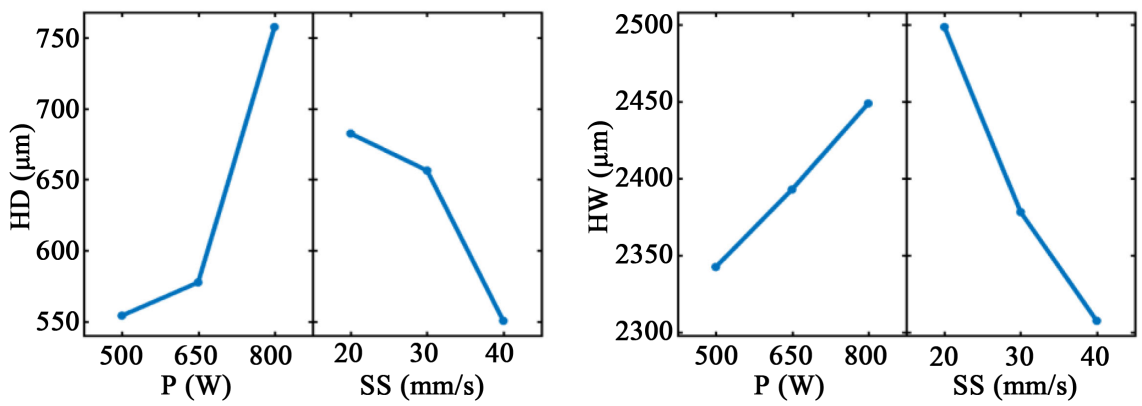

Figure 10. Mains effects of laser parameters on HD and HW for trochoid moving pattern.

Table 7. Correlation analysis between laser parameters and hardened zone dimensions for the considered scanning patterns.

\begin{tabular}{|c|c|c|c|c|c|c|c|c|c|}
\hline & & \multicolumn{2}{|c|}{ Linear } & \multicolumn{2}{|c|}{ Sinusoidal } & \multicolumn{2}{|c|}{ Triangular } & \multicolumn{2}{|c|}{ Trochoid } \\
\hline & & HD & $\mathrm{HW}$ & $\mathrm{HD}$ & $\mathrm{HW}$ & HD & $\mathrm{HW}$ & HD & $\mathrm{HW}$ \\
\hline Power & & $88.43 \%$ & $65.50 \%$ & $67.28 \%$ & $39.51 \%$ & $71.52 \%$ & $32.28 \%$ & $76.29 \%$ & $47.59 \%$ \\
\hline Scanning & peed & $45.49 \%$ & $69.52 \%$ & $70.79 \%$ & $89.68 \%$ & $64.17 \%$ & $92.91 \%$ & $49.48 \%$ & $85.48 \%$ \\
\hline \multirow{2}{*}{ Linear } & HD & - & $90.79 \%$ & $92.56 \%$ & $75.21 \%$ & $92.91 \%$ & $70.12 \%$ & $89.72 \%$ & $81.46 \%$ \\
\hline & HW & $90.79 \%$ & - & $95.48 \%$ & $85.24 \%$ & $91.24 \%$ & $84.02 \%$ & $93.33 \%$ & $92.35 \%$ \\
\hline \multirow{2}{*}{ Sinusoidal } & HD & $92.56 \%$ & $95.48 \%$ & - & $87.56 \%$ & $96.41 \%$ & $84.38 \%$ & $84.25 \%$ & $96.39 \%$ \\
\hline & HW & $75.21 \%$ & $85.24 \%$ & $87.56 \%$ & - & $83.26 \%$ & $98.10 \%$ & $73.03 \%$ & $93.34 \%$ \\
\hline \multirow{2}{*}{ Triangular } & HD & $92.91 \%$ & $91.24 \%$ & $96.41 \%$ & $83.26 \%$ & - & $80.32 \%$ & $79.98 \%$ & $91.89 \%$ \\
\hline & HW & $92.91 \%$ & $84.02 \%$ & $84.38 \%$ & $98.10 \%$ & $80.32 \%$ & - & $71.15 \%$ & $91.16 \%$ \\
\hline \multirow{2}{*}{ Trochoid } & HD & $92.91 \%$ & $84.02 \%$ & $84.25 \%$ & $73.03 \%$ & $79.98 \%$ & $71.15 \%$ & - & $77.33 \%$ \\
\hline & HW & $81.46 \%$ & $92.35 \%$ & $96.39 \%$ & $93.34 \%$ & $91.89 \%$ & $91.16 \%$ & $77.33 \%$ & - \\
\hline
\end{tabular}

ANOVA is also used to develop linear multiple regression models in order to establish a simplified empirical relationship to estimate or predict the hardened depth and the hardened width as a function of the laser parameters for the four moving patterns. Table 8 presents the achieved regression models for both HD and HW that include the process parameters and their interactions. Curves shown in Figures 11-14 present comparisons between measured and predicted hardened depth and hardened width. The predicted HD and HW values follow the measured values with an overall error of less than $10 \%$.

Applying the same experimental design for the four scanning patterns provides the possibility to evaluate their direct effects on the hardened depth and the hardened width. The experimental results reveal that linear scanning pattern achieves the maximal HD and the minimal HW compared to other scanning patterns. In the case of test 7 with a laser power of $800 \mathrm{~W}$ and a scanning speed of $20 \mathrm{~mm} / \mathrm{s}$, the linear pattern produces a hardened zone characterized by $\mathrm{HD} / \mathrm{HW}$ of $1011 / 1154 \mu \mathrm{m}$, while the sinusoidal pattern produces a $\mathrm{HD} / \mathrm{HW}$ of $456 / 2808 \mu \mathrm{m}$. Under the same conditions and for the same test, the triangular 

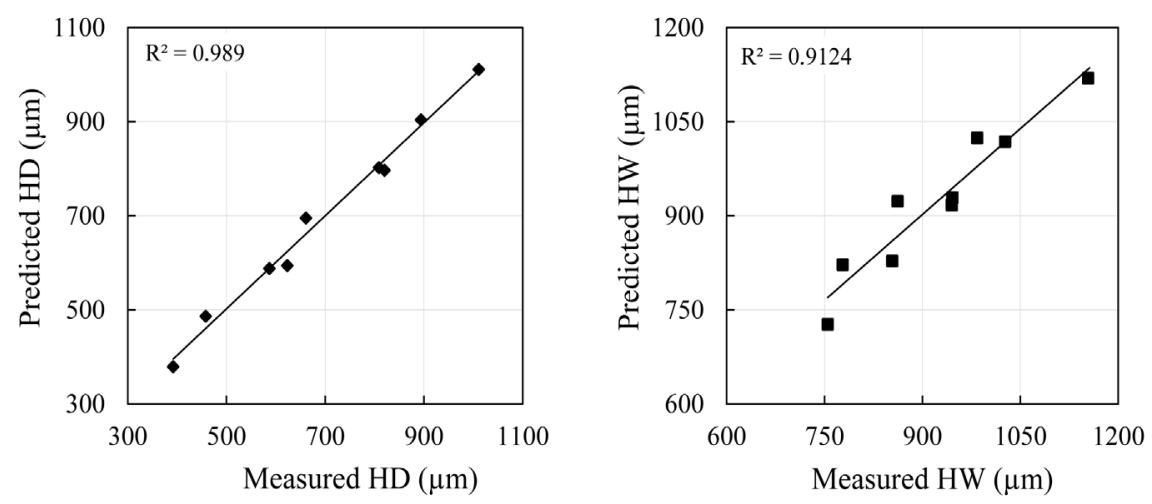

Figure 11. Scatter plot-Measured and predicted HD and HW for linear scaning pattern.
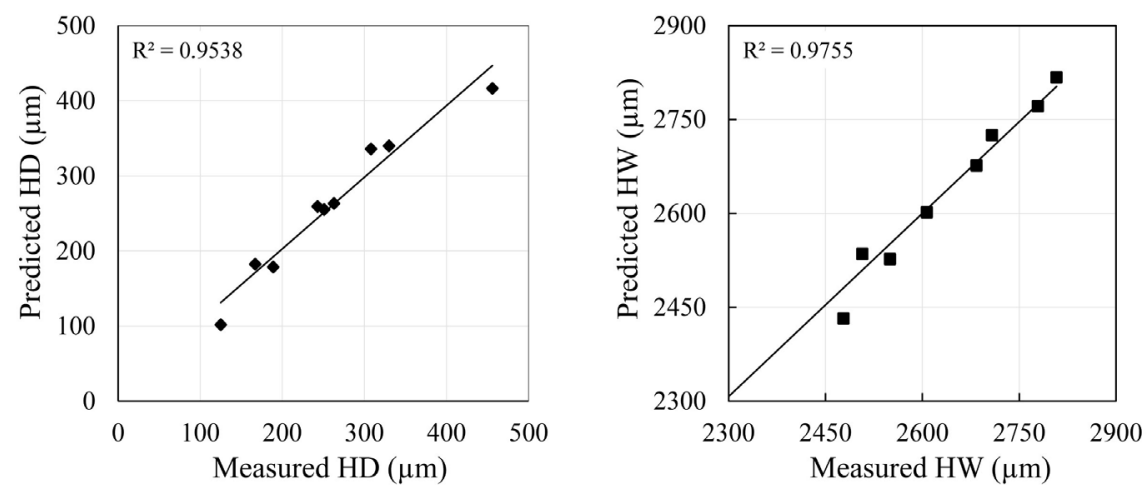

Figure 12. Scatter plot-Measured and predicted HD and HW for sinusoidal scaning pattern.
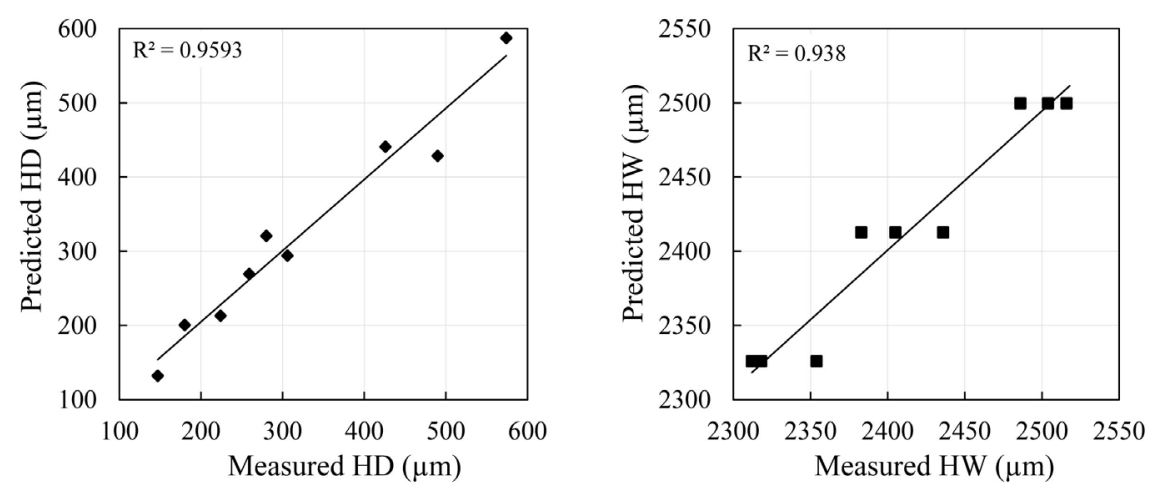

Figure 13. Scatter plot-Measured and predicted HD and HW for triangular scaning pattern.

Table 8. Regression equations derived from ANOVA for the four moving patterns.

\begin{tabular}{ccc}
\hline Patterns & Hardened depth & Hardened width \\
\hline Linear & $\mathrm{HD}=243+1.19 \mathrm{P}-15.07 \mathrm{SS}+0.006 \mathrm{P} * \mathrm{SS}$ & $\mathrm{HW}=755+0.724 \mathrm{P}-8.1 \mathrm{SS}-0.003 \mathrm{P} * \mathrm{SS}$ \\
Sinusoidal & $\mathrm{HD}=-49+0.846 \mathrm{P}-0.81 \mathrm{SS}-0.011 \mathrm{P} * \mathrm{SS}$ & $\mathrm{HW}=3157-0.07 \mathrm{P}-29.3 \mathrm{SS}+0.019 \mathrm{P} * \mathrm{SS}$ \\
Triangular & $\mathrm{HD}=-383+1.58 \mathrm{P}+6.13 \mathrm{SS}-0.026 \mathrm{P} * \mathrm{SS}$ & $\mathrm{HW}=2631+0.05 \mathrm{P}-10.6 \mathrm{SS}+0.004 \mathrm{P} * \mathrm{SS}$ \\
Trochoid & $\mathrm{HD}=605+0.34 \mathrm{P}-13.8 \mathrm{SS}+0.011 \mathrm{P} * \mathrm{SS}$ & $\mathrm{HW}=2269+0.63 \mathrm{P}-3.48 \mathrm{SS}-0.009 \mathrm{P} * \mathrm{SS}$ \\
\hline
\end{tabular}



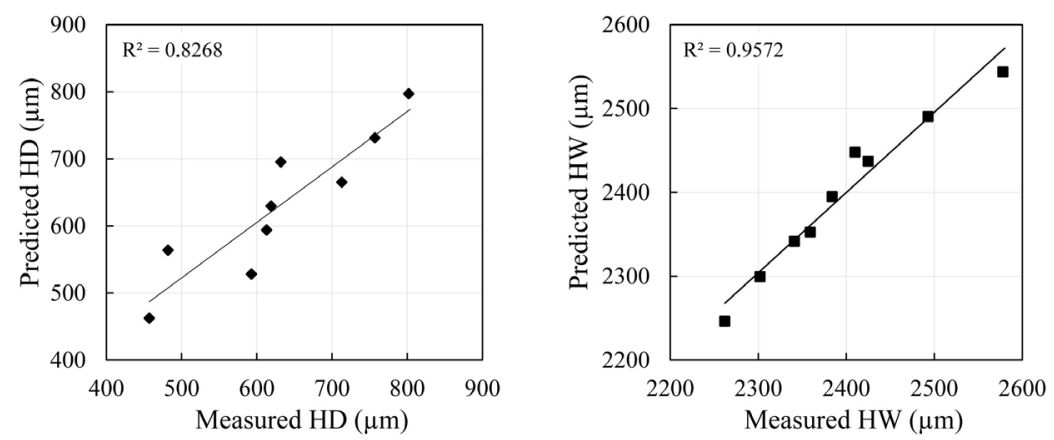

Figure 14. Scatter plot-Measured and predicted HD and HW for trochoid scaning pattern.

trajectory reaches an $\mathrm{HD} / \mathrm{HW}$ of $574 / 2516 \mu \mathrm{m}$, while the $\mathrm{HD} / \mathrm{HW}$ of the trochoid trajectory reaches $802 / 2578 \mu \mathrm{m}$. Figure 15 presents an overview of the effect of each moving pattern on HD and HW. Figure 16 presents the micro-hardness measurement results in the form of hardness curves produced for test 7 achieved using maximum power and minimum scanning speed for each scanning pattern. The hardness curves confirm the same tendencies observed in Figure 15. When using a sinusoidal scanning pattern, the laser beam spends much more time in the peripheral areas of the surface to be treated compared to the triangular or trochoid pattern, which concentrates more time and power in the central zone that increases the hardened depth. Although the trochoid pattern presents good result, it generates a non-uniform HD. This lack of uniformity can cause several problems in the case of thin steel parts surface heat treatment.

When the dimensions of the surface to be treated are larger than the cross section of the laser beam, some specific scanning patterns can be used to overcome the problem caused by the multi-track approach. The objective of choosing these scanning patterns is to maximize the surface of the hardened zone. This consists of maintaining a maximum uniform hardened width while respecting a minimum limit of hardened depth. In this perspective, if a minimum threshold is set for HD, the HW/HD ratio can be used as an indicator to evaluate the hardened zone surface according to the laser parameters for the different scanning patterns. HW/HD ratio also provides useful information on how heat is absorbed by the surface. A priori, if HW/HD increases, the hardened surface increases on condition that HD is kept close to HD limit. Table 9 presents the HW/HD ratio for various combinations of laser parameters and scanning patterns. The global average HW/HD ratio is, of course, higher than the average calculated excluding tests where HD is lower than $200 \mu \mathrm{m}$. In this table, it can be observed that sinusoidal and triangular scanning patterns present the highest HW/HD ratio. Therefore, these two patterns allow to achieve the largest hardened surfaces. With high HD and small $\mathrm{HW}$, the linear pattern produces the low and relatively constant $\mathrm{HW} / \mathrm{HD}$ ratio.

Although producing a higher ratio and therefore a higher HW than linear pattern, the trochoid pattern presents practically constant HW/HD ratio. These observations are confirmed by the contour plot presented in Figure 17. These 


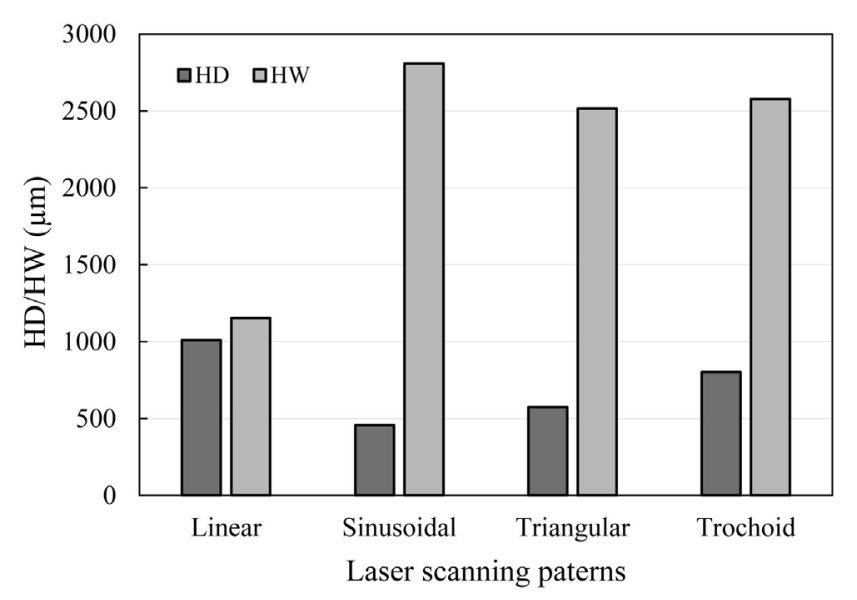

Figure 15. Comparison of $\mathrm{HD}$ and $\mathrm{HW}$ achieved using various scanning patterns.

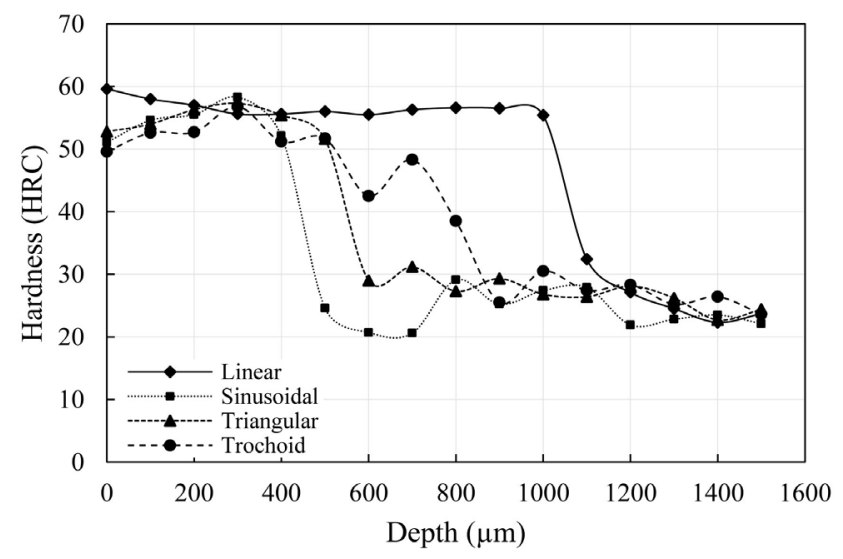

Figure 16. Micro-hardness measurements achieved using the considered scanning patterns (test 7).

Table 9. HW/HD ratio for various combinations of laser parameters and scanning patterns.

\begin{tabular}{|c|c|c|c|c|c|c|}
\hline \multirow{2}{*}{ Tests } & \multicolumn{2}{|c|}{ Laser parameters } & \multicolumn{4}{|c|}{$\mathrm{HW} / \mathrm{HD}$ ratio for scanning patterns } \\
\hline & Power & Scanning speed & Linear & Sinusoidal & Triangular & Trochoid \\
\hline 1 & 500 & 20 & 1.52 & 10.30 & 9.60 & 3.96 \\
\hline 2 & 500 & 30 & 1.86 & $\underline{15.27}$ & $\underline{13.36}$ & 3.95 \\
\hline 3 & 500 & 40 & 1.93 & $\underline{18.34}$ & $\underline{15.73}$ & 4.95 \\
\hline 4 & 650 & 20 & 1.22 & 8.42 & 5.11 & 3.94 \\
\hline 5 & 650 & 30 & 1.30 & 10.73 & 8.70 & 3.85 \\
\hline 6 & 650 & 40 & 1.33 & $\underline{13.11}$ & 10.51 & 4.78 \\
\hline 7 & 800 & 20 & 1.14 & 6.16 & 4.38 & 3.21 \\
\hline 8 & 800 & 30 & 1.15 & 8.71 & 5.83 & 3.18 \\
\hline 9 & 800 & 40 & 1.15 & 9.99 & 7.74 & 3.31 \\
\hline \multicolumn{3}{|c|}{ Average } & 1.40 & 11.23 & 9.00 & 3.90 \\
\hline \multicolumn{3}{|c|}{ Average excluding $\mathrm{HD}<200 \mathrm{~mm}$} & 1.40 & 9.05 & 7.41 & 3.90 \\
\hline
\end{tabular}



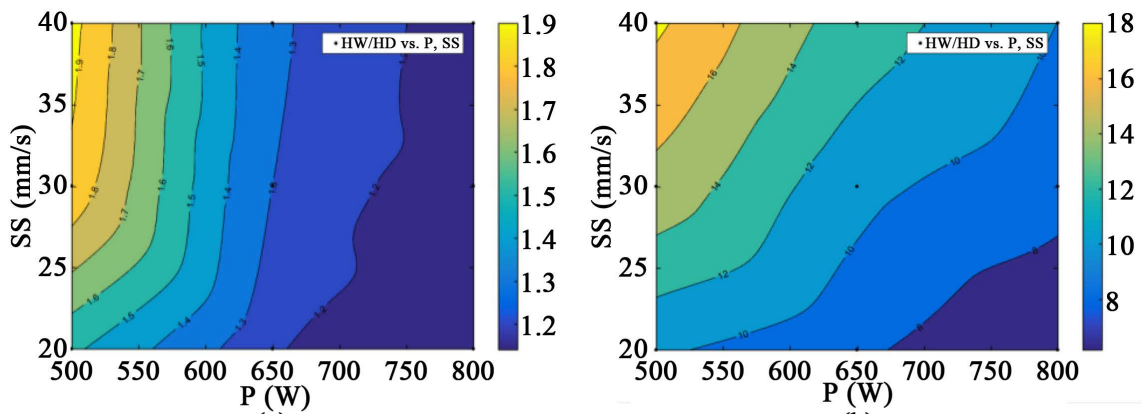

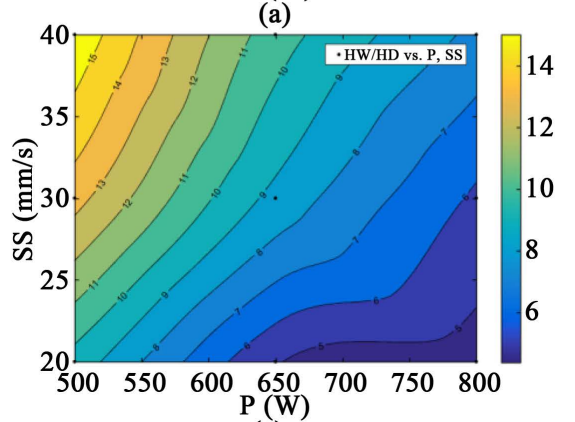

(c)

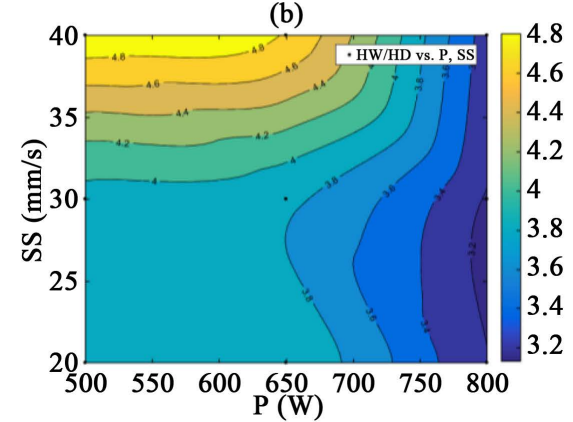

(d)

Figure 17. Contour plot of HW/HD ratio vs P and SS for (a) linear; (b) sinusoidal; (c) triangular and $(\mathrm{d})$ trochoid scanning pattern.

graphs reveal that $\mathrm{HW} / \mathrm{HD}$ ratio increases with SS and decreases with $\mathrm{P}$. This is typical because HW/HD tends to growth when HD decreases. If the laser parameters can be set to control a constant minimal HD close to $200 \mu \mathrm{m}$, by adopting the maximum ratio, HW can reach a minimum of 385, 3670, 3145 and 990 $\mu \mathrm{m}$ for linear, sinusoidal, triangular and trochoid scanning pattern, respectively. If the total generated heating energy is adjusted to maintain a constant minimal HD, HW can reach higher values when using sinusoidal or triangular patterns.

\section{Conclusion}

This paper presents an experimental investigation of laser surface hardening of AISI 4340 steel using different laser scanning patterns. The experimentations are carried out using a $3 \mathrm{~kW} \mathrm{Nd}$ : YAG laser system and considering four laser source moving patterns: linear, sinusoidal, triangular and trochoid pattern. The effects of laser process parameters, such as laser power and scanning speed and their interactions on the hardened depth and hardened width, for various laser scanning patterns, are evaluated and analyzed using structured experimental design and improved statistical analysis tools. Graphs of main effects, percent contributions, F-test, regression analysis, correlation analysis and response surface methodology are programmed, implemented and discussed for each scanning pattern. The results show that the laser power positively affects the hardened depth more than the hardened width, while the scanning speed negatively affects the hardened depth but more significantly the hardened width. However, the parameter's interactions have little effect on the hardness profiles. The results show also that the sinusoidal pattern gives a maximum width with an acceptable 
depth and a uniform hardness distribution, whereas the linear pattern gives a maximum depth with a width. The trochoid pattern presents a relatively good compromise between hardened depth and hardened width, but with an irregular hardness distribution and a non-uniform hardened depth. Hence, if a maximum of hardened width is required, it is recommended to use the sinusoidal pattern. For an extensive evaluation of the laser scanning pattern effects, it will be interesting to develop a numerical predictive model to conduct numerical investigations using a $3 \mathrm{D}$ thermal analysis.

\section{Conflicts of Interest}

The authors declare no conflicts of interest regarding the publication of this paper.

\section{References}

[1] Shercliff, H. and Ashby, M. (1991) The Prediction of Case Depth in Laser Transformation Hardening. Metallurgical Transactions A, 22, 2459-2466. https://doi.org/10.1007/BF02665012

[2] Selvan, J.S., Subramanian, K. and Nath, A.K. (1999) Effect of Laser Surface Hardening on En18 (AISI 5135) Steel. Journal of Materials Processing Technology, 91, 29-36. https://doi.org/10.1016/S0924-0136(98)00430-0

[3] Ashby, M. and Easterling, K.E. (1984) The Transformation Hardening of Steel Surfaces by Laser Beams-I. Hypo-Eutectoid Steels. Acta Metallurgica, 32, 1935-1948. https://doi.org/10.1016/0001-6160(84)90175-5

[4] Leung, M.K.H., Man, H.C. and Yu, J.K. (2007) Theoretical and Experimental Studies on Laser Transformation Hardening of Steel by Customized Beam. International Journal of Heat and Mass Transfer, 50, 4600-4606. https://doi.org/10.1016/j.ijheatmasstransfer.2007.03.022

[5] Steen, W., Watkins, K.G. and Mazumber, J. (2010) Laser Material Processing. 4th Edition, Springer, London, xvii, 558 p.

[6] Mioković, T., Schulze, V., Vöhringer, O. and Löhe, D. (2006) Prediction of Phase Transformations During Laser Surface Hardening of AISI 4140 Including the Effects of Inhomogeneous Austenite Formation. Materials Science and Engineering, 435-436, 547-555. https://doi.org/10.1016/j.msea.2006.07.037

[7] Lakhkar, R.S., Shin, Y.C. and Krane, M.J.M. (2008) Predictive Modeling of Multi-track Laser Hardening of AISI 4140 Steel. Materials Science and Engineering, 480, 209-217. https://doi.org/10.1016/j.msea.2007.07.054

[8] Martínez, S., Lesyk, D., Lamikiz, A., Ukar, E. and Dzhemelinsky, V. (2016) Hardness Simulation of Over-Tempered Area during Laser Hardening Treatment. Physics Procedia, 83, 1357-1366. https://doi.org/10.1016/j.phpro.2016.08.143

[9] Woodard, P.R. and Dryden, J. (1998) Thermal Analysis of a Laser Pulse for Discrete Spot Surface Transformation Hardening. Journal of Applied Physics, 85, 2488-2496. https://doi.org/10.1063/1.369610

[10] Xue, L., Islam, M.U. and McGregor, G. (1999) Dot Matrix Hardening of Steels Using a Fiber Optic Coupled Pulsed Nd:YAG Laser. Materials and Manufacturing Processes, 14, 53-65. https://doi.org/10.1080/10426919908914804

[11] Straffelini, G., Menapace, C. and Molinari, A. (2002) Interpretation of Effect of Ma- 
trix Hardening on Tensile and Impact Strength of Sintered Steels. Powder Metallurgy, 45, 167-172. https://doi.org/10.1179/003258902225002587

[12] Farrahi, G.H. and Sistaninia, M. (2009) Thermal Analysis of Laser Hardening for Different Moving Patterns. International Journal of Engineering Transactions A: Basics, 22, 169-180. http://inis.iaea.org/search/search.aspx?orig_q=RN:40101143

[13] Zhou, H. (2011) Temperature Rise induced by a Rotating or Dithering Laser Beam. Advanced Studies in Theoretical Physics, 5, 443-468.

[14] Komanduri, R. and Hou, Z.B. (2001) Thermal Analysis of the Laser Surface Transformation Hardening Process. International Journal of Heat and Mass Transfer, 44, 2845-2862. https://doi.org/10.1016/S0017-9310(00)00316-1

[15] Komanduri, R. and Hou, Z.B. (2004) Thermal Analysis of Laser Surface Transformation Hardening-Optimization of Process Parameters. International Journal of Machine Tools and Manufacture, 44, 991-1008. https://doi.org/10.1016/j.ijmachtools.2004.01.011

[16] Sistaninia, M. (2009) Laser Heat Treatment of Metals Using Rotating and Dithering Beams. In: Recent Advances in Technologies, IntechOpen. https://doi.org/10.5772/7396

[17] Tan, T. and Zhou, H. (2013) Numerical Solution for a Transient Temperature Distribution on a Finite Domain Due to a Dithering or Rotating Laser Beam. International Journal of Operations Research and Information Systems, 4, 38. https://doi.org/10.4018/ijoris.2013100102

[18] Philip, J.R. (1995) Taguchi Techniques for Quality Engineering. McGraw-Hill, New York, 249. 